\title{
La Oligoepilepsia. ¿Una Manera Diferente de Ser Epiléptico?
}

\author{
Dr. Marcelo Devilat B. ${ }^{1}$; Dr. Peđro Menéndez G. ${ }^{2}$.
}

\section{Oligoepilepsy}

Two patients with oligoepilepsy are presented. A girl, in her third year of life had an epileptic attack of the atonic type: In the following year she suffered one generalized tonic epileptic crisis and in her ninth year of life a Grand tmal seizure; her EEG was normal. The second is the case of a boy, who, in his sixth year of tife suffered an atonic epileptic crisis with slonical discharges: This seizure pattern recurred three years later; his EEG showed difuse slowness in the basal rhythm. In both children personal and family history and physical examination were normal. All their crisis lasted short times and no antiepileptic diug treatment was prescribed to them. The authors propose to name "oligoepilepsy" those epileptic seizures without well known trigger mechanisms, and a frequency not excecding one seizure per year and three in 4 to 5 years. Treatment should be used only when one or more of the following characteristics is present:Crisis more than 15 minutes long. focal scizures, organic brain damage, irritative potential in the EEG tracing; antecedents of epilepsy among fïrst degree relatives.

(Key words: Oligoepilepsy. Seizures. Fpilepsy. Ocassional convulsions).

Las crisis epilépticas, es decir, aquellos episodios de origen cerebral que resultan de una descarga neuronal excesiva, paroxística y sincrónica, manifestados por alteraciones de la conciencia y/o síntomas motores, sensoriales, psíquicos 0 autonómicos ${ }^{1} \cdot 2$, son frecuentes en la poblacion general. Entre el 4 y $6 \%$ de todas las personas han sufrido por lo menos una crisis epileptica en su vida ${ }^{3} \cdot \mathbf{4}$.

Entre las crisis epilépticas existen algunas cuyo origen tiene una cusa aguda -como intoxicaciones, trastomos metabólicos o enfermedades meningoencefálicas-. Son las llamadas "crisis ocasionales" y por lo tanto no constituyen epilepsia ${ }^{5}-5$.

Otto grupo de crisis epilépticas ocurre en ausencia de procesos agudos y pueden presentarse en tres formas diferentes: Como epilepsia propiamente tal, con crisis recurrentes, repetitivas y frecuentes 1 - 2 ; Como crisis aislada que aparece por primera y última vez, constituyendo precisamente las llamadas "crisis únicas" $2 \cdot 7-8$. ${ }^{9}$, o bien, entre ambos extremos en una tercera forma con episodios infrecuentes o que se repiten muy alejadamente, dando origen a la denominada "Oligoepilepsia"5-6:10-11-12.

Se presentan dos casos de oligoepilepsia, apro-

- Servicio de Neurología y Psiquiatría. Hospital Luis Calvo Mackenna. Departamento de Pediatría. Facultad de Medicina. División Oriente. Universidad de Chiłe.

2 Departamento de Neurología Infantil. Hospital San Juan de Dios. Facultad de Medicina. División Occidente. Universidad de Chile. vechando para hacer una revisión de la literatura sobre el tema e intentar una delimitación del cuadro.

\section{Casos Clínicos}

L.P.L. nacida el 23 de Diciembre de 1972. Presentó a los 3 años de edad pérdida brusca de conciencia, hipotonía generalizada, retroversión ocular, se recuperó en pocos minutos y luego durmió varias horas; No consultó. Un año después, mientras jugaba, cayó al suelo inconciente, con retroversión ocular e hipertonía generalizada, relajación esfinteriana y pocos minutos después había recuperado el conocimiento. Tratada de inmediato con Fenobarbital intramuscular, durmió algunas horas; Tampoco consultó esta vez. A los 9 años de edad, mientras se duchaba, sufrió pérdida del conocimiento, convulsiones tónicoclónicas generalizadas, trismus y retroversión ocular; El episodio duró algunos minutos, al cabo de los cuales durmió varias horas, despertando con cefalea. Antecedentes personales, familiares $y$ examen físico no fueron relevantes; Electroencefalograma (EEG) normal. Se diagnosticó oligoepilepsia, se dejó sin tratamiento, no volvió a control.

A.V.M. Nacido el 10 de Octubre de 1972, a los 6 años de edad perdió la conciencia y cayó al suelo con hipotonía generalizada, espuma por la boca, retroversión ocular y relajación esfinteriana; 7 minutos después se había recuperado, permaneciendo por algunos momentos algo confuso; No consultó. A los 9 años de edad, al quedarse dormido sufrió una crisis semejante. 
Los antecedentes personales, familiares, y el examen físico no mostraron nada notable. El EEG reveló algunos ritmos theta con tendencia paroxística bilateral. Se djagnosticó oligoepilepsia y no se indicó tratamiento. Se controló 10 meses después comprobándose que había estado libre de crisis.

\section{COMENTARIO}

En estos dos casos las crisis epilépticas fueron generalizadas desde el comienzo, no hubo paresia postictal, la duración fue inferior a los 15 minutos, el EEG resultó no irritativo y la frecuencia de las crisis fue muy escasa. Ninguno de los enfermos recibió tratamiento.

En la práctica casos como estos no son infre. cuentes de observar. Muchas veces, en los antecedentes familiares de los epilépticos aparece la información de algún pariente que nunca se hizo tratamiento a pesar de haber tenido crisis epilepticas infrecuentemente.

Es sotprendente que no se le haya dado la debida importancia a esta forma de presentación de las crisis epilépticas y no exista información acerca de su incidencia y prevalencia ${ }^{5}$. Una destacada publicación, como el Diccionario de la Epilepsia ${ }^{1}$, no menciona la oligoepilepsia, tampoco la literatura norteamericana. La Comisión que revisó la nueva clasificación de las crisis epilépticas $^{13}$ tampoco nombra a esta entidad.

Algunos autores europeos se han preocupado del tema. En efecto, Matthes ${ }^{5}$ define a la oligoepilepsia como aquellas "epilepsias que sin tratamiento especifico se manifiestan como una o pocas crisis epilépticas o bien crisis yue ocurren en un limitado período de la vida". Esta definición es, sin embargo, contradictoria pues si hay una crisis aislada, no es posible, en rigor, hablar de epilepsia ${ }^{2} 2$ y por otra parte no señala cuantas crisis se espera que ocuran en el periodo mencionado de la vida, ni cual es la duración del mismo. Los conceptos de Matthes ${ }^{5}$ son sin embargo de utilidad, pues como se observa en los casos 1 y 2, se enfatiza acerca de "pocas erisis ocurridas en un limitado periodo de la vida como puede ser la infancia".

Degen ${ }^{6}$ considera que se debe hablar de oligoepilepsia cuando una persona tiene crisis parciales de Gran mal cada 2 ó 3 años. Surge así el concepto de tipo y frecuencia de las crisis. Desde este punto de vista, los enfermos de los casos 1 y 2 cumplen con los requisitos de Degen para ser incluidos como oligoepilépticos.

Para Doose ${ }^{10}$ el concepto de oligoepilepsia debe circunscribirse a crisis generalizadas desde el comienzo, afebriles, que no ocurren más de 2 veces por año. Aparece en este autor el criterio de crisis libres de fuctores desencadenantes, como fiebre y excluyendo cualquier episodio de origen focal. Los pacientes de esta comunicación cumplen los criterios de Doose, de no tener factores desencadenantes y sufrir crisis generalizadas desde el comienzo, pero el mismo autor agrega en su definición que los episodios ocurren entre los 9 a los 16 años de cdad. Con lo que ambos casos quedarían excluidos. Sin embargo, Doose ${ }^{10}$ no da razones para los limites temporales que esta. blece, de modo que parece licito seguir incluyendo a nuestros pacjentes en el concepto de oligoepilepsia.

Por último, para Kellermann ${ }^{12}$, oligoepilepsia no es sino tener menos de 2 crisis epilépticas al an̄o.

Como se puede apreciar no existe entre los pocos autores que se han dedicado al tema, uniformidad acerca de to que debe llamarse oligoepilepsia.

Fs evidente que un concepto claro acerca de este tipo de crisis permitirá tener una acertada conducta terapéutica. Por una parte, se evitará tratar sin necesidad al paciente, evitando exponerlo a los riesgos de los anticonvulsivantes y a los problemas psicosociales que origina el tratamiento prolongado $15 \cdot 16 \cdot 17 \cdot 18 \cdot 19 \cdot 20 \cdot 21$ - 22 y por otra, no se dejará sin medicamentos a otros enfermos que realmente los requieran.

Llegados a este punto, uno se debería preguntar, cuándo sc trata una oligoepilepsia y cuales podrian ser los factores de predicción de nuevas crisis.

Kellermann ${ }^{12}$ sostiene que una paresia postictál es indicación suficiente para iniciar tratamiento, pero no da las razones que lo inclinan por tal actitud. La paresia postictal se observa infrecuentemente y en ausencia de indicadores de organicidad, es expresión de agotamiento neuro. nal transiturio, y no necesariamente predictor de nuevas crisis. El mismo autor aconscja tratar a aquellos enfermos cuyos hallazgos clínicos y de laboratorio indiquen que se espera uл aumento en la frecuencia de las crisis, sin especificar cuales son dichos predictores ${ }^{12}$.

Por su parte, Matthes ${ }^{5}$ piensa que deberían ser tratadas las oligoepilepsias con más de una crisis por año, siempre que los factores desencadenantes como la falta de sueño, el alcoholismo etc., hubiesen sido evitadas, sin tratar as crisis infre. cuentes que ocurren durante el sueño.

A este respecto, Doose ${ }^{10}$ es más liberal, opinando que los pacientes portadores de oligoepilepsias constituidas por Gran mal generalizado desde el comienzo, cuyas crisis aparezcan con una frecuencia de 46 más al año, deberian ser medicados, siempre que se hayan evitado los factores desencadenantes. Este autor va un poco 
más lejos y piensa que si bien las crisis del tipo Petit mal deben ser tratadas, aquellos episodios constituidos por ausencias simples en la pubertad $y$ que ocurren infrecuentemente en un enfermo o que no lo molesten, podrían ser dejados en observación y no tratarse. Es más, aunque Doo. $\mathrm{se}^{10}$ no incluye específicamente a las crisis focales dentro de la oligoepilepsia, aconseja que estos episodios podrían no tratarse si ocurren tan infrecuentemente como menos de 2 al año.

Es evidente que enfermos portadores de crisis infrecuentes como $l$ al año, podrían permanecer sin medicación como se observa en los casos de esta publicación. Por otra parte, como se destaca en $\operatorname{los}$ casos 1 y 2, los mismos pacientes o sus familiares no se interesan por recibir tratamiento si los episodios son infrecuentes. Otro asunto es cuando el paciente sufre varias crisis epilépticas al año. EI enfermo y su familia dificilmente tolerarían quedar sin tratamiento en esas circunstancias.

Es difícil estar con Doose ${ }^{10}$ en relación a su criterio con respecto a las ausencias simples que aparecen en la pubertad. Es cierto que no siempre éstas se presentan en la forma "Pyknolepti. ca", es decir, todos los días, pero incluso en la forma cíclica, las crisis pueden ocurrir varias veces al día ${ }^{23}$ por semanas y meses, lo cual puede interferir en la vida psíquica del paciente y lo expone, según el efecto "Kindling"'24 a Status de ausencia ${ }^{23-25-26}$. También estamos en desacuerdo con la proposición de no tratar las crisis focales, si se presentan infrecuentemente ${ }^{10}$. Es cierto que la forma Centroparietal focal de la Epilepsia Rolándica es benigna y que algunos autores no siempre indican medicamentos en ellas $^{27-28-29}$, pero las crisis focales, aunque muchas veces son funcionales, por lo menos en los niños, requieren por su especial evolución $\mathrm{y}$ pronóstico, una cuidadosa atención aunque ocurran infrecuentemente ${ }^{30-31}$. Además, las epilep. sias focales que han estado largos años en tratamiento tienen un significativo peor pronóstico que las epilepsias generalizadas $31-32-33$. Por esto las crisis focales, aunque sean infrecuentes no deberían ser incluidas en las oligoepilepsias y naturalmente deberian ser tratadas.

Desde otro punto de vista, vale la pena señalar que los enfermos presentados en esta publicación no tenian antecedentes de epilepsia en sus familias, ni historia ni sjgnos sospechosos de organicidad. Estas variables deben ser tomadas en cuenta por cuanto los niños con daño de primera neurona, asi como los hijos y hermanos de padres epilépticos tienen mayores riesgos que la población general de sufrir epilepsia ${ }^{34-35-36}$. Por lo anterior, es recomendable tratar a aquellos enfer- mos oligoepilépticos que tengan las caracteristicas mencionadas.

El último elemento importante a considerar es el EEG. Para algunos autores el EEG no es un predictor destacado de nuevas crisis epilérticas en la oligoepilepsia, si se considera que en los niños el EEG está en relación a su maduración funcional ${ }^{10}$. Para otros autores, que se han preocupado del tema, un EEG irritativo (espiga, poliespiga seguidas o no de onda lenta) es sufjciente indicación como para iniciar el tratamien. to medicamentoso. Por otra parte y aunque el EEG en reposo e hiperventilación del tipo irritativo se puede encontrar en niños normales, en porcentajes que van del 1,1 al $3,5 \% 34 \cdot 35 \cdot 36$, existen opiniones que aconsejan tratar con antiepilépticos a los enfermos que presenten al menos 1 crisis epiléptica, con este tipo de alteraciones ${ }^{5-19}$, criterio este último, que no es compartido por otros investigadores ${ }^{2-11}$. Los casos presentados en esta publicación tuvieron EEG sin características irritativas, razón por la cual fueron dejados sin tratamiento.

Como puede observarse, no existe en la literatura, de por sí escasa, un criterio uniforme con respecto al concepto de oligoepilepsia, los factores predictores y la conducta terapéutica. Es posible que sea más práctico, circunscribir en la definición sólo el concepto de frecuencia de crisis y especificar cuales son las características del cuadro que podrían aconsejar iniciar un tratamiento antiepiléptico.

Proponemos llamar oligoepilepsia a las crisis epilépticas que, sin ser ocasionales ni tener evidentes factores desencadenantes, ocurran con una frecuencia no mayor de una al año ni de tres en 4 ó 5 años. Los enfermos con esta modalidad de presentación, no deberían ser tratados a menos que tengan una $o$ más de las siguientes características: Episodio que dure más de 15 minutos: crisis focales o de inicio focal; Antece. dente de epilepsia en familiares de primer grado (padres y hermanos); Signos de daño orgánico ( $\mathrm{Cl}$ inferior a 70), signos de lesión de primeta neurona $o$ ambos y EEG irritativo.

\section{RESUMEN}

Se presentan dos niños portadores de oligoepi* lepsia. Se comentan las características clínicas y EEG del problema. Se propone denominar oligoepilepsia a las crisis epilèpticas que sin ser ocasionales ni tener evidentes factores desencadenantes, ocurran con una frecuencia no mayor que una vez al año ni mayor que tres episodios en 4 ó 5 años. Se discuten las condiciones bajo las que debiera instalarse un tratamiento anticonvulsivo en estos pacientes. 


\section{REFERENCLAS}

1 Gostout, H.: Diccionatio de epilepsia. Parte ]: Defīniciones. Organización Mundial de la Salud, Suiza, 1973.

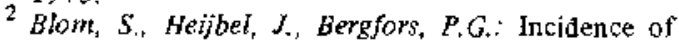
epilepsy in children: a follow-up study thrce years after the fjrst seizure. Epilcpsia 19: 343, 1978.

${ }^{3}$ Crombie, D.L., Cross, K.W. Fry, J., Pinset, R.J.F.H.: A survey of the epilepsies in general practice. A report by the Research Commitee of the College of General Practitioners. Brit. Med, J. 6: 416, 1960.

4 W.H.O.: Kpilepsy. WId. Hlth. Org. Techn. Rep. Ser, No 130, 1957.

5 Matrhes A.: lipilepsie. Diagnostik und Therapie für Klinik und Praxis. Thicme, Stuttgart, 1977, S, 122 If. unđ $83 \mathrm{f}$.

6 Degen, R.: Die Kindlichen Anfallsteiden. Kipileptische und nichtepileptische Anfälle. Hippokrates, Stuttgart, 1976, S. 69-71.

'Thomas, M.H.: The single seizure: its study and managemen1. J.A.M.A. 169:457, 1959.

8 Saunders, $M$. and Marshall, $C_{\text {. }}$ : Isolated seizures; an ElGG and clinical assesment. Epilepsia 16: 731, 1975.

9 Devilat, M. Menéndez, $P$., Chamorro, R., Acevedo. C.: Seguimiento de niños con una ctisis epiléptica no tratada, Rev. Chil. Pediatr. 55: 9, 1984

10 Doose, $H$. .: Indikationen zur linleintung und Beendigung ciner antiepileptischen Therapie im Kindesalter. In: Kruse, R. (Hrsg): Epilepsie. Thieme, Sttutgart, 1971, S. 9-15.

11 Jonz, D.: Indikationen zur Finleitung und Beendigung einer antiepilcptischen Therapic bei Jungendlichen und Erwachsenen. In: Kruse, R. (Hisg): tpi* lepsie. Thieme, Stuttgart, 1971, S. 15-19.

12 Kellermann, $K$.: Therapie der Epilepsie in Kindesalter. Dtsch. med. Wschr. 103: 492, 1978.

13 Bancaud, $J$. , Henriksen, O, Rubio-Donnadieu, F., Masekatsu, $S$., Dreifuss, $F . E$., Penry. K.: Proposal for revised clinical and electroencephalographic classificatıon of epileptic seizures. Fpilepsia 22: 489, 1981.

14 Janz, $D$. In tema di remissione e di ricomparsa de crisi durantc e dopo il trattamento farmacológíco dellepilessia. Boll, Lega It. Epil. 39: 95, 1982.

15 Reynolds $_{s}$ E.H.: Chronic antiepileptic toxicity. A review. Epilepsia 16:319, 1975.

16 Srores, G.: Schoolchildten with epilepsy at risk for learning and behaviour problems. Develop. Med. Child Neurol. 20: 502, 1978.

17 Trimble, $M$. Corbert, $J$.: Anticonvulsant drugs and congnitive function. In: Wada, J.A. and Penry, J.K. (cds): Advances in Epileptiology. Raven Press, New York, 1980, pp. 113.

18 Magendzo, S., Devilat M., A staburkoga, I., Mena, P.. Rauch, M.: Opinión acerca de epilepsia en tres grupos de madres y los efectos que tiene la educación. Rev. Chil. Pcdiatr. 50: 87, 1979.

19 Ryan, R., Kempner, $K$., Emlen, A.: The stigma of epilepsy as self-concepto. Epilepsia 21: 433, 1980.

20 Word, F. Bower, B.: A study of certain social aspects of epilepsy in childhood. In: Jones, M.O. (Ed): Kipilepsy 78. The British Epilepsy Association, Londo, 1978, pp.27-34
21 Hoppe, $R$. Die Epilepsie aus sozial medizinischer Sicht. Fortschr. Neurol. Psychiat. 48: J01, 1980.

22 Mariuca, $N$.: Lfectos de los anticonvulsivantes sobre el cocficiente intelectual. Servicio de Neurología $y$ Psiquiatría. Hospital Luis Całvo Mackenna. Santiago de Chile (Comunicación persona))

23 Janz, D.: Die Epilepsien. Spezielle Pathologie und Therapie. Thieme, Suttgart, 1969. S. 77 und 127.

24 Livingston, $\boldsymbol{X}$ : Kindline- a new exprimental model of epilepsy. In: Jones, M.O. (Ed.): Fpilepsy 79. The British Epilepsy Association, London, 1979, pp. 55-.59.

2s Andermann, F., Robh, J.P.: Absence Status. A reapprajal following review of 38 patients. Fipilepsia 13: 177,1972 .

20 Christion, $\boldsymbol{W}$.: Statusformen kleiner cpileptischer Anfälle, Nervenarzt $\$ 1: \$ 91,1980$.

27 Loiseau, $P$, Cohadon, F, Mortureoux, $Y .:$ A propos d'une forme singuliete d'epilepsie de L'enfant. Rev. Neurol. 116: 244, 1967 .

28 Becumanoir, A., Bollis, $T$, Varfis, $G$, Ansari, $K$. Benign apilepsy of childhood with Rolandic spikes. Epilepsia 15: 301, 1974,

29 Laman, P. Kivity, S.: Bening focal epilepsy of childhond. Arch. Neurol. 32: 261, 1975.

${ }^{30}$ Roger, J., Dravet, C., Menendez, P, Btireau. $M_{*}$ : Les epilepsies partielles de l'entant - evolution et facteurs de pronostic. Rev. F.F.G. Neurophysiol. 1]: 431,1981 .

31 Holbowach, J., Thurston, D.L., Hixon, B.B., Keller, A.J.: Prognosis in childhood epilepsy. Additional follow-up of 148 childen 15 to 23 years after withdrawal of anticonvulsant therapy. New Fingl. J. Med. 306: 831, 1982.

32 Groh, C: Zur Frage der Heilbarkeit kindlicher Epilepsion. Wien Klin. Wochensehr. 87: 1, 1975.

33 Hollowach. J., Thurston, D.L., OLeary, I.: Prognosis in childhood epilepsy. Follow-up study of 148 cases in twhich therapy had been suspended after prolonged anticonvulsant control. New Fngl. J. Med. 286: 169, 1972.

34 Latorre, K.. Devilat, M., Valdivieso, M.A.: Daǹo neucológico cn el nirio, aspectos clínicos y ctiológicos (I). Rev. Chil. Pediatr. 44: 237, 1973.

${ }^{3} J_{a n z}, D$. Scheffner. D.: Uber epileptische Anfälle hei Kinder von Eltem mit Epilepsie. Kervenarat 51: 226 , 1980.

36 Metrakos, K., Metrakos, J.D.: Genetics of convalsive disorders. II. Genetcs and electroencephalographic studies in centrencephalic epilepsy. Neurology 11 : 414, 1961

${ }^{37}$ EEG-Olofsson, O., Petersen, I., Sellden, U.: The development of the $E E G$ in normal children from age of ] through 15 years. Neuropäđiatrje 2: 375 , $19 ? 1$.

3 Dowse, H. Gerken, H, Hien-Volpel, K.F., Volzke, $E_{*}$ : Genetics of photosensitive epilepsy. Neuropädiatrie 1: 56,1969 .

${ }^{39}$ Gibhs, F.A. Cribhs, E.L.: Atlas of Electroencephalography. Addison and Wesley, Cambridge, Mass. 1964. vol. III pp. 538.

40 Cavazztit G.B., Capella, L. Nalin, A.: Longitudinal study of epileptiform El:G patterns in normal children. Fpilepsia 21: 43, 1980. 